\title{
Fetal Brain Anomalies Associated with Ventriculomegaly or Asymmetry: An MRI-Based Study
}

\author{
(DE. Barzilay, (D) Bar-Yosef, (D) D. Dorembus, (D) R. Achiron, and (DE. Katorza
}

\begin{abstract}
BACKGROUND AND PURPOSE: Fetal lateral ventriculomegaly is a relatively common finding with much debate over its clinical significance. The purpose of this study was to examine the association between ventriculomegaly and asymmetry and concomitant CNS findings as seen in fetal brain MR imaging.
\end{abstract}

MATERIALS AND METHODS: Fetal brain MR imaging performed for various indications, including ventriculomegaly, with or without additional ultrasound findings, was assessed for possible inclusion. Two hundred seventy-eight cases found to have at least 1 lateral ventricle with a width of $\geq 10 \mathrm{~mm}$ were included in the study. Ventriculomegaly was considered mild if the measurement was $10-11.9 \mathrm{~mm}$; moderate if, 12-14.9 mm; and severe if, $\geq 15 \mathrm{~mm}$. Asymmetry was defined as a difference of $\geq 2 \mathrm{~mm}$ between the 2 lateral ventricles. Fetal brain MR imaging findings were classified according to severity by predefined categories.

RESULTS: The risk of CNS findings appears to be strongly related to the width of the ventricle (OR, 1.38; 95\% Cl, 1.08-1.76; $P=.009)$. The prevalence of associated CNS abnormalities was significantly higher $(P=.005)$ in symmetric ventriculomegaly compared with asymmetric ventriculomegaly (38.8\% versus $24.2 \%$, respectively, for all CNS abnormalities and $20 \%$ versus $7.1 \%$, respectively, for major CNS abnormalities).

CONCLUSIONS: In this study, we demonstrate that the rate of minor and major findings increased with each millimeter increase in ventricle width and that the presence of symmetric ventricles in mild and moderate ventriculomegaly was a prognostic indicator for CNS abnormalities.

$\mathbf{F}$ etal lateral ventriculomegaly is a relatively common finding. Some have estimated the incidence of ventriculomegaly identified on sonography to be about $1 \% .{ }^{1}$ Fetal ventriculomegaly is defined as a dilation of the lateral ventricle atrium to a width of $\geq 10 \mathrm{~mm} .{ }^{2,3}$ A measurement of $10-12 \mathrm{~mm}$ is commonly referred to as mild ventriculomegaly, while measurements of 12-15 and $>15 \mathrm{~mm}$ are defined as moderate and severe ventriculomegaly, respectively. ${ }^{4}$ Severe ventriculomegaly has been associated with poorer neurodevelopmental outcome compared with mild ventriculomegaly ${ }^{5,6}$; however, it has been previously suggested that the neurodevelopmental outcome in cases of moderate ventriculomegaly is similar to that observed in mild ventriculomegaly. ${ }^{7}$

Received July 9, 2016; accepted after revision September 19.

From the Department of Obstetrics and Gynecology (E.B., R.A., E.K.) and Pediatric Neurology Unit (O.B.-Y.), Sheba Medical Center, Tel-Hashomer, Ramat-Gan, Israel; and Sackler School of Medicine (E.B., O.B.-Y., S.D., R.A., E.K.), Tel Aviv University, Tel Aviv, Israel.

E. Barzilay and O. Bar-Yosef contributed equally to this work.

Please address correspondence to Eran Barzilay, MD, PhD, Department of Obstetrics and Gynecology, Sheba Medical Center, Tel-Hashomer, Ramat-Gan, 52621, Israel; e-mail: eran.barzilay@gmail.com

http://dx.doi.org/10.3174/ajnr.A5009
Many studies have focused on the neurodevelopmental outcome of children who were diagnosed in utero with lateral ventriculomegaly. ${ }^{6,8-16}$ One of the most important factors determining outcome is the presence and severity of additional CNS anomalies. Although most anomalies can be identified by using sonography, MR imaging was shown to be superior in identifying CNS anomalies. A recent study found that MR imaging could detect additional findings in $15.3 \%$ of the seemingly isolated ventriculomegaly/ventricle asymmetry cases. ${ }^{17}$ In this study, we aimed to establish the proportion of MR imaging-detected CNS anomalies associated with ventriculomegaly in correlation with the severity of ventriculomegaly and ventricle asymmetry. To prevent underestimation of associated anomalies, we did not exclude cases with CNS anomalies previously identified on sonography. To the best of our knowledge, this is the first large-scale MR imaging study that has examined the association between lateral ventricle width and asymmetry and CNS anomalies.

\section{MATERIALS AND METHODS}

We conducted a cross-sectional study of all brain MR imaging scans performed in a single tertiary center (Sheba Medical Center, Tel-Hashomer, Israel) between January 2011 and December 2014. 


\begin{tabular}{|c|c|c|c|}
\hline \multirow{2}{*}{$\begin{array}{l}\text { Malformation } \\
\text { Type }\end{array}$} & \multicolumn{3}{|c|}{ Severity } \\
\hline & Normal Variant & Minor Findings & Major Findings \\
\hline NTD & & Meningocele & $\begin{array}{l}\text { Acrania/anencephaly encephalocele } \\
\text { Myelocele }\end{array}$ \\
\hline \multirow[t]{3}{*}{ Cystic lesions } & CPC & Isolated PVPC & Nonisolated PVPC \\
\hline & Cavum verge & Arachnoid cyst & PVL \\
\hline & CVI & & Porencephalic cyst \\
\hline Bleeding & & IVH grade 1 & $\mathrm{IVH}$ grade $2-4$ \\
\hline Ischemia & & & Parenchymatic damage \\
\hline \multirow[t]{4}{*}{ Cortical disorders } & Delayed sulcation (up to 2 weeks) & & Lissencephaly \\
\hline & & & Schizencephaly \\
\hline & & & Heterotopia \\
\hline & & & Microcephaly \\
\hline White matter & Isolated T2 hypersignal & & Nonisolated T2 hypersignal \\
\hline Midline CC & Lipoma & $\begin{array}{l}\text { Short intact CC } \\
\text { thick/thin }\end{array}$ & Complete/partial CC agenesis \\
\hline Midline CSP & & Isolated septal agenesis & SOD \\
\hline \multirow[t]{4}{*}{ Posterior fossa } & MCM & Blake pouch cyst & Chiari 2 \\
\hline & & & DWS \\
\hline & & & $\begin{array}{l}\text { Primary disruption of } \\
\text { cerebellum/vermis/brain stem }\end{array}$ \\
\hline & & & $\begin{array}{l}\text { Secondary disruption of } \\
\text { cerebellum/vermis/brain stem }\end{array}$ \\
\hline Vascular & & & Vein of Galen aneurysm \\
\hline
\end{tabular}

Note:-NTD indicates neural tube defect; CPC, choroid plexus cyst; CVI, cavum vellum interpositum; PVPC, periventricular pseudocyst; PVL, periventricular leukomalacia; IVH, intraventricular hemorrhage; CMV, cytomegalovirus; CC, corpus callosum; CSP, cavum septum pellucidum; SOD, septo-optic dysplasia; MCM, mega cysterna magna; DWS, Dandy-Walker syndrome; CSVT, cerebral sinovenous thrombosis.

"Ventriculomegaly" was defined as a lateral ventricle width of $\geq 10 \mathrm{~mm} \cdot{ }^{2-4}$ Lateral ventricle asymmetry was defined as a difference of $\geq 2 \mathrm{~mm}$ between the 2 lateral ventricles. All MR imaging scans during the study period were assessed for possible eligibility, regardless of the reason for the referral. Inclusion criteria for this study were singleton pregnancy and ventriculomegaly (at least 1 lateral ventricle with a width of $\geq 10 \mathrm{~mm}$ ). Exclusion criteria were fetal cytomegalovirus or toxoplasma infection and cases with abnormal genetic tests (karyotype or microarray). Two hundred seventy-eight cases met the inclusion/exclusion criteria.

Fetal MR imaging was performed with a 1.5T system (Optima 1.5T; GE Healthcare, Milwaukee, Wisconsin) as previously published. ${ }^{17}$ Single-shot fast spin-echo T2-weighted sequences in 3 orthogonal planes were performed by using the following parameters: section thickness, 3-4 mm; no gap; flexible coil (8-channel cardiac coil); matrix, $320 \times 224$; TE, $90 \mathrm{~ms}$; and TR, $1298 \mathrm{~ms}$. The FOV was determined by the size of the fetal head: $24 \mathrm{~cm}$ for the smaller fetuses and $30 \mathrm{~cm}$ for the larger fetuses. T1 fast-spoiled gradient-echo sequences were performed only in the axial plane with a larger FOV (400 mm), with section thickness, $4 \mathrm{~mm}$; gap, $0.5 \mathrm{~mm}$; TR, $160 \mathrm{~ms}$; and TE, $2.3 \mathrm{~ms}$. Measurements of the lateral ventricle widths were obtained on the coronal plane at the level of the ventricles (with good visibility of the choroid plexuses). For this study, ventriculomegaly was considered mild if the measurement was 10-11.9 mm; moderate if, $12-14.9 \mathrm{~mm}$; and severe if, $\geq 15 \mathrm{~mm}$. Asymmetry was defined as a difference of $\geq 2 \mathrm{~mm}$ between the 2 lateral ventricles.

Fetal brain MR imaging findings were classified according to severity by predefined categories as shown in the Table. Classification of findings into minor or major was determined on the basis of previously published data. ${ }^{18-27}$

Statistical analyses were conducted by using the Statistical
Package for the Social Sciences (SPSS, Version 23; IBM, Armonk, New York). Q-Q plots were used to assess normality. Continuous variables were described as mean (SD) or median (interquartile range) as appropriate. Continuous variables were compared by using an unpaired $t$ test and a 1-way ANOVA test as appropriate or by using the Mann-Whitney $U$ test and Kruskal Wallis test for nonparametric continuous variables as appropriate. The $\chi^{2}$ test was used for comparison of categoric variables. Logistic regression analysis was used to examine the relationship among ventricle width, width difference, and CNS findings. Significance was $P<.05$.

The study was approved by the local institutional review board of the Sheba Medical Center.

\section{RESULTS}

Two hundred seventy-eight pregnancies were included in the study. The average maternal age was $31.8 \pm 5.2$ years. Maternal age did not differ significantly between cases with or without ventricle asymmetry (32 versus 31.4 years, respectively; $P=.693$ ) or among cases of mild, moderate, and severe ventriculomegaly (32, 31.5 , and 29.5 years of age, respectively; $P=.235$ ). The median gestational age at MR imaging acquisition was 32 weeks (interquartile range, 31-34 weeks). There was no difference in median gestational age between cases with or without ventricle asymmetry (32 versus 32 weeks, respectively; $P=.079$ ) or among cases of mild, moderate, and severe ventriculomegaly $(32,32$, and 31.5 weeks, respectively, $P=.785)$.

Median ventricle width for the widest ventricle was $11 \mathrm{~mm}$ (interquartile range, $11-13 \mathrm{~mm}$ ), and for the contralateral ventricle, it was $9 \mathrm{~mm}$ (interquartile range, $7-11 \mathrm{~mm}$ ). Median ventricle width difference was $3 \mathrm{~mm}$ (interquartile range, 1-4 mm).

Figure 1 depicts the distribution of ventriculomegaly cases according to severity and the presence or absence of asymmetry. 
Most cases were defined as mild ventriculomegaly (73\%), while moderate and severe ventriculomegaly comprised $22.7 \%$ and $4.3 \%$ of the cases, respectively. Most cases of ventriculomegaly were asymmetric $(71.2 \%)$.

Figure 2 depicts the distribution of CNS findings according to the width of the ventricle (the larger of the 2 ventricles). The risk of CNS findings appears to be strongly related to the width of the ventricle. The association between CNS findings and ventricle width was particularly evident in moderate ventriculomegaly, in which each 1-mm increase in ventricle width increases the risk for both minor and major CNS findings. Logistic regression analysis, with each ventricle width along with maternal age, gestational age at the time of MR imaging, and fetal sex as variables, demonstrated that the widths of both ventricles were positively associated with an increased risk for CNS anomalies (OR, 1.38; 95\% CI, $1.08-1.76 ; P=.009$, for the maximal ventricle; and OR, $1.21 ; 95 \%$ CI, $1.03-1.44 ; P=.025$, for the contralateral ventricle). Gestational age at the time of MR imaging was found to be negatively

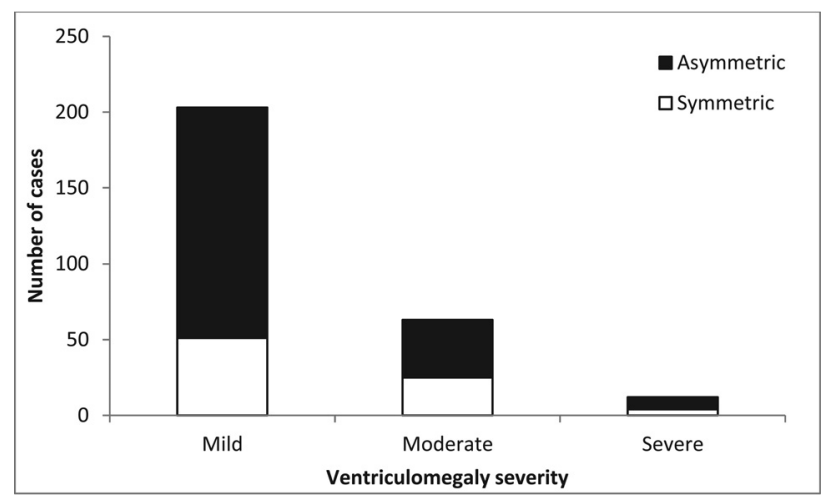

FIG 1. Distribution of ventriculomegaly cases according to severity and the presence or absence of asymmetry. Filled bars represent asymmetric ventriculomegaly; empty bars represent symmetric ventriculomegaly.

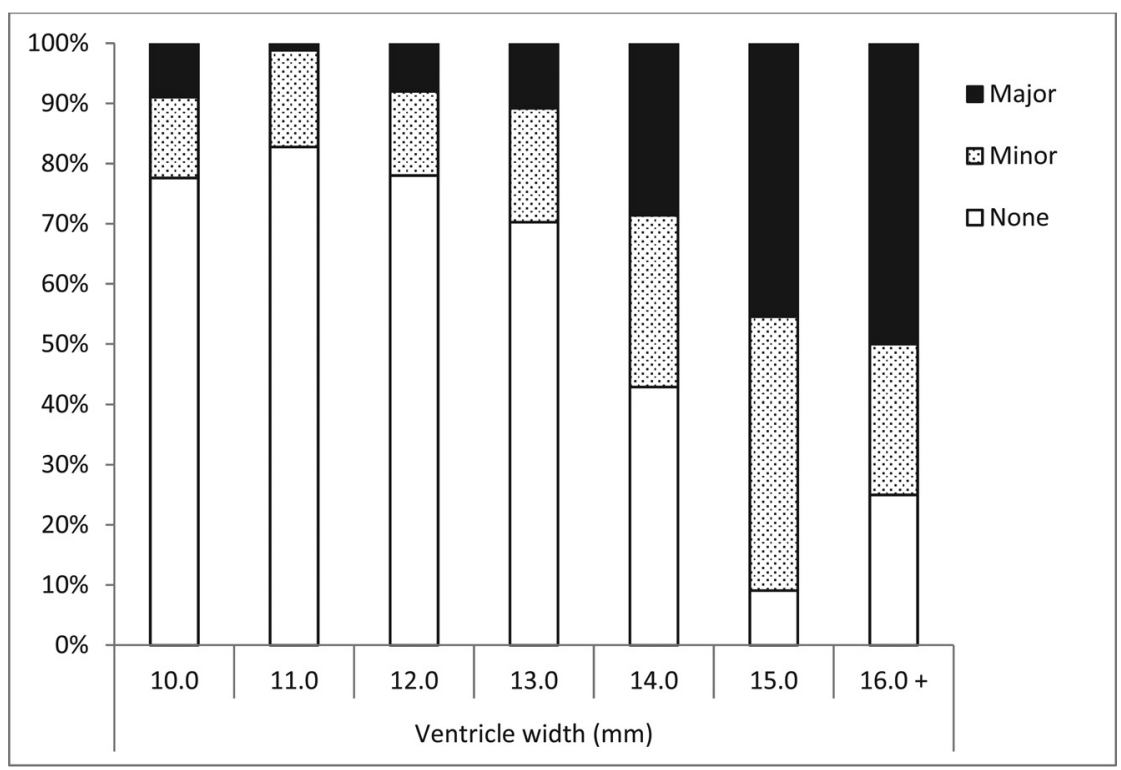

FIG 2. Distribution of additional CNS findings in cases of fetal ventriculomegaly according to maximal ventricle width. Filled bars represent major CNS findings, dotted bars represent minor CNS findings, and empty bars represent no additional CNS findings. Due to small numbers, cases with dilation of $\geq 16 \mathrm{~mm}$ were grouped together. associated with CNS anomalies (OR, 0.86; 95\% CI, 0.77-0.95; $P=.003)$, possibly indicating that the more complicated cases tended to be diagnosed earlier. Maternal age $(P=.75)$ and fetal sex $(P=.304)$ were not found to be associated with CNS anomalies. The correlation between ventricle width and associated anomalies appears to be strongest within the 12- to $15-\mathrm{mm}$ range because the OR for CNS anomalies increased from 1.38 (95\% CI, $1.08-1.76 ; P=.009)$ to 2.53 (95\% CI, $1.48-4.34 ; P=.001)$ when the analysis was restricted to cases with a ventricle width of $12-15$ $\mathrm{mm}$.

Most cases in this study were asymmetric ventriculomegaly (71\%). We have found more CNS abnormalities in symmetric ventriculomegaly compared with asymmetric ventriculomegaly (38.8\% versus $24.2 \%$, respectively) and more major CNS abnormalities in symmetric ventriculomegaly compared with asymmetric ventriculomegaly ( $20 \%$ versus $7.1 \%$, respectively). These differences between symmetric and asymmetric ventriculomegaly were found to be statistically significant $(P=.005)$.

Figure 3 illustrates the distribution of CNS abnormalities according to ventriculomegaly severity and the presence or absence of asymmetry. We found that the differences in the risk of CNS abnormalities between symmetric and asymmetric ventriculomegaly were only apparent in the mild and moderate groups. In cases of severe ventriculomegaly, the rates of minor $(25 \%)$ and major (50\%) findings were identical in symmetric and asymmetric ventriculomegaly. The number of cases in the severe ventriculomegaly group $(n=12)$ was much smaller than in the moderate $(n=63)$ and mild $(n=203)$ groups.

Next, we used regression analysis to explore the individual impact of ventricle width and ventricle asymmetry on the risk of CNS findings. Using maximal ventricle width and width difference as variables, we found that ventricle width was an independent risk factor for CNS findings (OR, 1.56; 95\% CI, 1.32-1.85; $P<.001)$, while ventricle width difference was inversely associated with CNS findings (OR, 0.79; 95\% CI, $0.68-0.92 ; P=.003)$. When average ventricle width was used in the regression model instead of maximal ventricle width, average ventricle width appeared to be independently associated with CNS findings (OR, 1.56; 95\% CI, 1.32 1.85 ; $P<.001)$. However, width difference lost its significant association with CNS findings (OR, 0.99; 95\% CI, $0.84-$ $1.16 ; P=.896)$.

\section{DISCUSSION}

In this study we analyzed a large number MR imaging scans of fetuses diagnosed with fetal ventriculomegaly. We have shown that the risk of minor and major CNS findings is closely related to the width of the lateral ventricle. The association between ventricle width and CNS findings is particularly evident in moderate ventriculomegaly. As seen in Fig 2, the rate of minor and major findings significantly increased with each millime- 


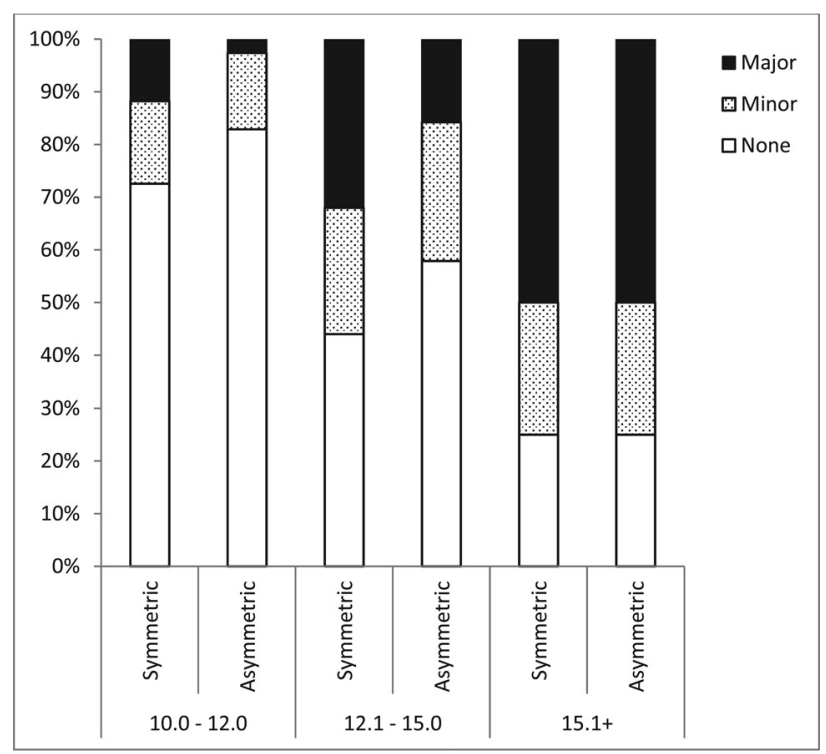

FIG 3. Distribution of additional CNS findings in cases of fetal ventriculomegaly according to ventriculomegaly severity and the presence or absence of asymmetry. Filled bars represent major CNS findings, dotted bars represent minor CNS findings, and empty bars represent no additional CNS findings.

ter increase in ventricle width. Thus, while the prevalent categorization of mild, moderate, and severe ventriculomegaly appears to adequately portray the risk of CNS anomalies, taking into account the ventricle width greatly improves the risk estimation. Moreover, these findings are contrary to those in previous studies that defined mild ventriculomegaly at an atrial width of 10-15 mm. ${ }^{7,16}$

Another intriguing result of this study is the correlation between ventricle asymmetry and CNS findings in fetal MR imaging. We have found that symmetric ventriculomegaly was associated with more CNS findings than asymmetric ventriculomegaly. This difference was apparent in mild and moderate ventriculomegaly, while the rates of minor and major CNS findings were similar between symmetric and asymmetric severe ventriculomegaly. The apparent advantage of asymmetric ventriculomegaly might be somewhat counterintuitive. However, the presence of asymmetry for a case with a given ventricle width translates to a smaller contralateral ventricle. Thus, in asymmetric mild ventriculomegaly, the contralateral ventricle will be in the normal range, while in moderate ventriculomegaly, most cases will have a contralateral ventricle width in the normal-to-mild ventriculomegaly range. This hypothesis gains support from our regression analysis that demonstrated that the association between width difference and CNS findings was eliminated when average ventricle width was used as a variable in the analysis instead of maximal ventricle width, thereby encompassing data on both ventricles.

The relatively low incidence of major anomalies in cases of mild symmetric ventriculomegaly $(2.6 \%)$ may merit re-evaluation of the cost-effectiveness of fetal MR imaging in these cases. Nevertheless, we believe that given the clinical implications of major CNS anomalies and in light of the prevalence of minor findings in mild asymmetric ventriculomegaly (14.5\%), fetal brain MR imaging still plays an important role in the work-up of these cases.

The data portrayed in this study aid in establishing the correlation between lateral ventricle width and CNS anomalies. The high rate of CNS anomalies observed in this study stresses the need for a thorough investigation of fetal ventriculomegaly and especially for meticulous examination in cases of moderate-tosevere ventriculomegaly and bilateral ventriculomegaly.

This study is in accordance with previous studies that found more CNS anomalies in moderate and severe ventriculomegaly compared with mild ventriculomegaly. ${ }^{10,11}$ However, in most of these studies, imaging was based almost exclusively on ultrasound, ${ }^{9-11}$ despite the known advantages of fetal brain MR imaging. ${ }^{17}$ Moreover, none of these studies, including those with fetal MR imaging as an imaging technique, ${ }^{15,17}$ have analyzed the impact of ventricle width on the risk of CNS anomalies.

One of the advantages of this study is that it included a large number of fetal MR imaging scans, enabling us to examine in depth the association between ventriculomegaly and CNS findings. Thus, we could identify differences in the rate of CNS findings not only among mild, moderate, and severe ventriculomegaly but also due to minute increases in ventricle width. Another major advantage is that the study is MR imaging-based, thereby increasing the ability to identify CNS abnormalities compared with neurosonography. ${ }^{17,28-31}$

This study is limited by the lack of data on neurodevelopmental outcome. Here we examined the association between ventriculomegaly and CNS anomalies in the fetus without assessing the outcome of the child. Nevertheless, because the presence of CNS abnormalities has been shown to be closely related to the neurodevelopmental outcome of the child, ${ }^{6}$ findings in fetal MR imaging can be used as an indication of the outcome. Moreover, to improve the clinical significance of the association between ventriculomegaly and CNS anomalies, we classified CNS anomalies according to severity (Table).

\section{CONCLUSIONS}

In this study, we demonstrate that the rate of minor and major findings increases with each millimeter increase in ventricle width and that the presence of symmetric ventricles in mild and moderate ventriculomegaly is a prognostic indicator for CNS anomalies in MR imaging examinations.

\section{REFERENCES}

1. Salomon LJ, Bernard JP, Ville Y. Reference ranges for fetal ventricular width: a non-normal approach. Ultrasound Obstet Gynecol 2007;30:61-66 CrossRef Medline

2. Cardoza JD, Goldstein RB, Filly RA. Exclusion of fetal ventriculomegaly with a single measurement: the width of the lateral ventricular atrium. Radiology 1988;169:711-14 CrossRef Medline

3. Almog B, Gamzu R, Achiron R, et al. Fetal lateral ventricular width: what should be its upper limit? A prospective cohort study and reanalysis of the current and previous data. J Ultrasound Med 2003;22: 39-43 Medline

4. International Society of Ultrasound in Obstetrics and Gynecology Education Committee. Sonographic examination of the fetal central nervous system: guidelines for performing the 'basic examination' and the 'fetal neurosonogram.' Ultrasound Obstet Gynecol 2007;29: 109-16 CrossRef Medline

5. Gaglioti P, Oberto $M$, Todros T. The significance of fetal ventriculomegaly: etiology, short- and long-term outcomes. Prenat Diagn 2009;29:381-88 CrossRef Medline

6. Weichert J, Hartge D, Krapp M, et al. Prevalence, characteristics and perinatal outcome of fetal ventriculomegaly in 29,000 pregnancies followed at a single institution. Fetal Diagn Ther 2010;27:142-48 CrossRef Medline

7. Melchiorre $\mathrm{K}$, Bhide $\mathrm{A}$, Gika $\mathrm{AD}$, et al. Counseling in isolated mild 
fetal ventriculomegaly. Ultrasound Obstet Gynecol 2009;34:212-24 CrossRef Medline

8. Mehta TS, Levine D. Imaging of fetal cerebral ventriculomegaly: a guide to management and outcome. Semin Fetal Neonatal Med 2005; 10:421-28 CrossRef Medline

9. Chu N, Zhang Y, Yan Y, et al. Fetal ventriculomegaly: pregnancy outcomes and follow-ups in ten years. Biosci Trends 2016;10:125-32 CrossRef Medline

10. Breeze AC, Alexander PM, Murdoch EM, et al Obstetric and neonatal outcomes in severe fetal ventriculomegaly. Prenat Diagn 2007;27: 124-29 CrossRef Medline

11. Gaglioti P, Danelon D, Bontempo S, et al. Fetal cerebral ventriculomegaly: outcome in $\mathbf{1 7 6}$ cases. Ultrasound Obstet Gynecol 2005;25:372-77 CrossRef Medline

12. Kelly EN, Allen VM, Seaward G, et al. Mild ventriculomegaly in the fetus, natural history, associated findings and outcome of isolated mild ventriculomegaly: a literature review. Prenat Diagn 2001;21: 697-700 CrossRef Medline

13. Laskin MD, Kingdom J, Toi A, et al. Perinatal and neurodevelopmental outcome with isolated fetal ventriculomegaly: a systematic review. J Matern Fetal Neonatal Med 2005;18:289-98 CrossRef Medline

14. Scala C, Familiari A, Pinas A, et al. Perinatal and long-term outcome in fetuses diagnosed with isolated unilateral ventriculomegaly: systematic review and meta-analysis. Ultrasound Obstet Gynecol 2016 Apr 19. [Epub ahead of print] CrossRef Medline

15. Ouahba J, Luton D, Vuillard E, et al. Prenatal isolated mild ventriculomegaly: outcome in 167 cases. BJOG 2006;113:1072-79 CrossRef Medline

16. Pagani G, Thilaganathan B, Prefumo F. Neurodevelopmental outcome in isolated mild fetal ventriculomegaly: systematic review and meta-analysis. Ultrasound Obstet Gynecol 2014;44:254-60 CrossRef Medline

17. Gat I, Hoffmann C, Shashar D, et al. Fetal brain MRI: novel classification and contribution to sonography. Ultraschall Med 2016;37: 176-84 CrossRef Medline

18. Toi A, Chitayat D, Blaser S. Abnormalities of the foetal cerebral cortex. Prenat Diagn 2009;29:355-71 CrossRef Medline

19. Santo S, D'Antonio F, Homfray T, et al. Counseling in fetal medicine: agenesis of the corpus callosum. Ultrasound Obstet Gynecol 2012;40: 513-21 CrossRef Medline

20. Vasudevan C, McKechnie L, Levene M. Long-term outcome of antenatally diagnosed agenesis of corpus callosum and cerebellar malformations. Semin Fetal Neonatal Med 2012;17:295-300 CrossRef Medline

21. Malinger G, Lev D, Ben Sira L, et al. Congenital periventricular pseudocysts: prenatal sonographic appearance and clinical implications. Ultrasound Obstet Gynecol 2002;20:447-51 CrossRef Medline

22. Epelman M, Daneman A, Blaser SI, et al. Differential diagnosis of intracranial cystic lesions at head US: correlation with CT and MR imaging. Radiographics 2006;26:173-96 CrossRef Medline

23. Volpe P, Campobasso G, De Robertis V, et al. Disorders of prosencephalic development. Prenat Diagn 2009;29:340-54 CrossRef Medline

24. Hayward R. Postnatal management and outcome for fetal-diagnosed intra-cerebral cystic masses and tumours. Prenat Diagn 2009; 29:396-401 CrossRef Medline

25. Carletti A, Colleoni GG, Perolo A, et al. Prenatal diagnosis of cerebral lesions acquired in utero and with a late appearance. Prenat Diagn 2009;29:389-95 CrossRef Medline

26. Garel C, Fallet-Bianco C, Guibaud L. The fetal cerebellum: development and common malformations. J Child Neurol 2011;26:1483-92 CrossRef Medline

27. Malinger G, Lev D, Lerman-Sagie T. The fetal cerebellum: pitfalls in diagnosis and management. Prenat Diagn 2009;29:372-80 CrossRef Medline

28. Morris JE, Rickard S, Paley MN, et al. The value of in-utero magnetic resonance imaging in ultrasound diagnosed foetal isolated cerebral ventriculomegaly. Clin Radiol 2007;62:140-44 CrossRef Medline

29. Benacerraf BR, Shipp TD, Bromley B, et al. What does magnetic resonance imaging add to the prenatal sonographic diagnosis of ventriculomegaly? J Ultrasound Med 2007;26:1513-22 Medline

30. Kandula T, Fahey M, Chalmers R, et al. Isolated ventriculomegaly on prenatal ultrasound: what does fetal MRI add? J Med Imaging Radiat Oncol 2015;59:154-62 CrossRef Medline

31. Salomon LJ, Ouahba J, Delezoide AL, et al. Third-trimester fetal MRI in isolated 10- to $12-\mathrm{mm}$ ventriculomegaly: is it worth it? $B J O G$ 2006;113:942-47 CrossRef Medline 